\title{
Frequency difference stabilization in Y-shaped cavity dual-frequency laser with the aid of precision passive control
}

\author{
Xiaofei Zeng, Bin Zhang,* Guangzong Xiao $\bullet^{*}$ * Jianping Liu, ${ }^{\dagger}$ \\ and Hongchang Zhao, \\ National University of Defense Technology, College of Advanced Interdisciplinary Studies, \\ Changsha, China
}

\begin{abstract}
We present a precise passive frequency difference stabilization scheme for the Y-shaped cavity dual-frequency laser. A two-stage thermostat and a precision steady current circuit were designed to stabilize the temperature and discharge current of the laser. The laser relative frequency difference drift rate is stabilized within $0.011 \%$ at room temperature. The result shows an improvement of two orders of magnitude over the stress-induced birefringence closed-loop control method. This scheme will further improve the accuracy of acceleration and precision force measurement. Moreover, the scheme shows the great potential in industrial applications because of the low cost and good environmental adaptability. In addition to the temperature change, we found that the mode repulsion also affects the stability of the frequency difference. (C) The Authors. Published by SPIE under a Creative Commons Attribution 4.0 Unported License. Distribution or reproduction of this work in whole or in part requires full attribution of the original publication, including its DOI. [DOI: 10.1117/1.OE.60.3.034107]
\end{abstract}

Keywords: dual-frequency laser; frequency difference drift; passive control; mode repulsion effect.

Paper 20201425 received Dec. 3, 2020; accepted for publication Feb. 22, 2021; published online Mar. 18, 2021.

\section{Introduction}

In recent years, the frequency difference stabilization of dual-frequency lasers has become important in the precise measurement fields for a variety of applications such as velocity and vibration measurement, ${ }^{1}$ high-resolution force sensing, ${ }^{2}$ laser lidar detection, ${ }^{3,4}$ feedback interferometers, ${ }^{5-7}$ acceleration measurement, ${ }^{8}$ atomic clocks, ${ }^{9}$ and Doppler velocimeters. ${ }^{10,11}$ In these applications, in addition to a large frequency difference tunable range, a low-frequency difference drift of the laser over a long period of time is generally required. For instance, the drift of the laser frequency difference may be required to be $<1 \mathrm{MHz}$ over hours of work in a long-term acceleration measurement. There are several methods that can be used to solve the problem of measurement error caused by frequency difference instability, and each has its advantages and disadvantages.

To meet the requirements of large frequency difference output, compact structure, and low cost, in 2011, we proposed an innovative Y-shaped cavity dual-frequency laser with an output frequency difference that can be continuously tuned in the range of 26 to $665 \mathrm{MHz}{ }^{12} \mathrm{~A}$ clever optical coating was designed to output both an orthogonally polarized $S$-light and $P$-light in one laser. One light is used as the reference light, and the other light is used as the signal light. The beat note of the two lights can be used for detecting interested physical quantities, such as force, ${ }^{13}$ acceleration, ${ }^{8}$ and so on. At present, its large frequency difference output, small size, and good acceleration and force measurement performance have been verified. ${ }^{8,12-14}$ To further improve the measurement accuracy, the stability of the frequency difference is crucial.

Many frequency difference stabilization methods are active and based on closed-loop feedback. ${ }^{15-18}$ On the one hand, although the accuracy of stability is high, most of them require bulky devices and complicated servo systems, leading to a high cost and limited stable frequency

*Address all correspondence to Bin Zhang, kd208zb@sina.com; Guangzong Xiao, xiaoguangzong@nudt.edu.cn

These authors contributed equally. 
difference. On the other hand, active frequency difference stabilization schemes limit the effectiveness of the measured frequency noise power spectrum to frequencies much higher than the servo bandwidth of the applied frequency lock. ${ }^{19}$ Blindly pursuing the accuracy of frequency stabilization will lose the significance of measuring frequency difference. In the Y-shaped cavity dual-frequency laser, one frequency was used as a reference and the other frequency as a signal. The relative frequency difference between the two was used to measure physical quantities. If the method of active stabilization is adopted, the two frequencies will be locked and unable to measure physical quantities. Therefore, according to the structural characteristics and working principle of the Y-shaped cavity dual-frequency laser, we decided to adopt a passive method to stabilize the frequency difference. The passive stabilization scheme has good short-term stability. If an ultra-low expansion coefficient cavity that realizes a vibration insensitive mount is used and the cavity temperature and discharge current stabilities are precisely controlled, then a long-term good frequency difference stability can be achieved. ${ }^{20-22}$

In this paper, we analyze the main factors that affect the frequency difference stability of the Y-shaped cavity dual-frequency laser. A precise passive control of the temperature and current scheme is proposed to reduce these influencing factors. After experimental verification, the relative frequency difference variation is $<80 \mathrm{kHz}$, and the relative frequency difference drift rate is $0.011 \%$, which is two orders of magnitude higher than the stress-induced birefringence closedloop control scheme. ${ }^{17}$ Also, it can be stabilized to any frequency difference in the output range. This cost-effective approach will improve the laser's potential in industrial applications.

\section{Theoretical Analysis}

The basic structure of the Y-shaped cavity dual-frequency laser is shown in Fig. 1.,12,13 The laser cavity is made up of ultra-low thermal coefficient $\left(1 \times 10^{-8}\right)$ Zerodur glass. Four optically contacted fused silica mirrors [M1, M2, M3, and polarization beam splitter (PBS)] were placed in the cavity. The reflectance of the plane output mirror M1 is $99.8 \%$. The reflectance of the concave reflective mirrors M2 and M3, with a radius of curvature $R=2 \mathrm{~m}$, is $99.99 \%$. The diameter of the mirrors is $9 \mathrm{~mm}$, and the thickness of the mirror is $4 \mathrm{~mm}$. The PBS has a special film design that is placed at the Brewster angle. It can transmit $99.9 \%$ of the $P$-light and reflect $99.9 \%$ of the $S$-light, so the $P$-light and $S$-light share gain and oscillate in different light paths. The $P$-light is the polarized light of the electric field vector that is perpendicular to the plane of incidence. The $S$-light is the polarized light of the electric field vector that is parallel to the plane of incidence. The polarization directions of the two are orthogonal. The common part of the two beams is called the common cavity. The gain tube is filled with helium-neon ( $\mathrm{He}-\mathrm{Ne}$ ) gas with a pressure ratio of $7: 1$ and $\mathrm{Ne}_{20}: \mathrm{Ne}_{22}=1: 1$. The independently used parts are called the $P$ sub-cavity and $S$ sub-cavity. Dry air at $101 \mathrm{kPa}$ fills both of them. Also, the sensing gas tube in the $P$ sub-cavity is filled with the same gas. It can be connected to the gas sylphon through a venthole. The pressure in sensing gas tube will change with the structure variation of gas sylphon and lead to the frequency difference change. The sensing gas can be replaced with any stable gas at any pressure to change the sensing sensitivity. The length of the common cavity is $73 \mathrm{~mm}$. So, the transit time between M1 and PBS is $2.44 \times 10^{-10} \mathrm{~m} / \mathrm{s}$. The lengths of the S sub-cavity and P sub-cavity are both $31.8 \mathrm{~mm}$. The piezoelectric transducer can adjust the cavity length to change the frequency difference. Under the effective combination of these factors, the stable oscillation of the dual-frequency laser with a wavelength of $632.8 \mathrm{~nm}$ is finally formed.

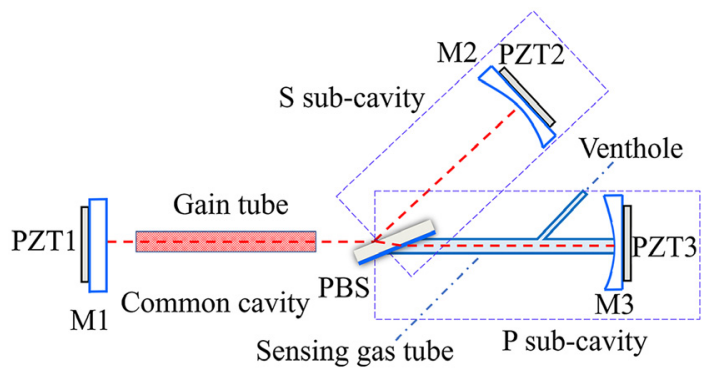

Fig. 1 The basic structure of the Y-shaped cavity dual-frequency laser. 
Compared with ordinary He-Ne dual-frequency lasers, the two sub-cavities of Y-shape cavity lasers are separated in space. The thermal behavior of the gain tube and the environmental temperature change causes different temperature gradients in the two sub-cavities. This is essential when considering the influence factors of the frequency difference fluctuation.

The laser theoretical model established by Lamb's optical maser theory has proven to be particularly effective for gas lasers in long-term practice. This theoretical analysis actually has little relation to the shape of the laser, but it has a close relation to the type of gain medium. According to the self-consistency equations of Lamb semi-classical theory, ${ }^{23}$ the laser frequencies of the $S$-light and $P$-light in two-mode operation are

$$
\begin{aligned}
& v_{S}=\Omega_{S}+\sigma_{S}+\rho_{S} E_{S}^{2}+\tau_{S P} E_{P}^{2} \\
& v_{P}=\Omega_{P}+\sigma_{P}+\rho_{P} E_{P}^{2}+\tau_{P S} E_{S}^{2}
\end{aligned}
$$

where the subscripts $S$ and $P$ represent the $S$-light and $P$-light, respectively, $\Omega_{n}(n=S, P)$ is the passive cavity resonance frequency, $\sigma_{n}$ is the frequency pulling parameter, $\rho_{n}$ is the self-pushing coefficient, $E_{n}^{2}$ is the light intensity, and $\tau_{n m}(n=S, P ; m=S, P)$ is the mutual pushing coefficient.

The output frequency difference of the dual-frequency laser is

$$
\begin{aligned}
\Delta v & =v_{P}-v_{S}=\left(\Omega_{P}-\Omega_{S}\right)+\left(\sigma_{P}-\sigma_{S}\right)+\left(\rho_{P} E_{P}^{2}+\tau_{P S} E_{S}^{2}-\rho_{S} E_{S}^{2}-\tau_{S P} E_{P}^{2}\right) \\
& =\Delta \Omega+\Delta v_{\sigma}+\Delta v_{E^{2}},
\end{aligned}
$$

It can be seen that the frequency difference of the Y-shaped cavity laser consists of three parts: (1) The passive cavity frequency difference term $\Delta \Omega$ represents the frequency difference of two orthogonally polarized lights in the passive cavity, which depends only on the cavity length difference of the two sub-cavities. (2) The mode-pulling effect term $\Delta v_{\sigma}$ represents the correction amount of the mode-pulling effect to the frequency difference. (3) The saturation effect term $\Delta v_{E}^{2}$ is composed of mutual pushing effect term and self-pushing effect term. Among them, $\Delta v_{\tau}=\tau_{P S} E_{S}^{2}-\tau_{S P} E_{P}^{2}$ is the mutual pushing effect term, which means the correction amount in the mode competition effect between two orthogonal polarization lights. $\Delta v_{\rho}=\rho_{P} E_{P}^{2}-\rho_{S} E_{S}^{2}$ is self-pushing effect term. Its influence on the laser frequency difference is opposite to the mode-pulling effect term. To simplify the theoretical derivation, it can be understood as weakening of the mode-pulling effect. At the same time, Ref. 23 pointed out that the influence of the self-repulsive term can be ignored in the case of a small signal, so simplified processing is feasible.

For the passive cavity frequency difference term $\Delta \Omega$, the main influencing factor is the change of the two sub-cavity lengths caused by mechanical vibration and temperature fluctuation. Taking temperature changes as an example, according to the laser resonance conditions, the change of the optical frequency difference of the $S$-light and $P$-light is obtained

$$
\Delta \Omega_{0}=\frac{q c\left(L \alpha_{1}+l \alpha_{2}\right) \Delta T}{2 L^{2}},
$$

where $q$ is a large integer, $c$ is the velocity of light, $L$ is the distance between the reflecting plates, $l$ is the thickness of PBS, $\alpha_{1}$ and $\alpha_{2}$ are the thermal expansion coefficient of the laser cavity material and PBS, respectively, and the temperature changes between the $S$ and $P$ sub-cavities is $\Delta T$. When the temperature changes by $1 \mathrm{mK}$, the corresponding frequency difference drift is $8.42 \mathrm{kHz}$.

For the mode-pulling effect term $\Delta v_{\sigma}$, the laser working material in the active cavity shows strong dispersion near the center frequency $v_{0}$ of the gain curve. When the gain coefficient is not zero, the refractive index is a function of frequency. ${ }^{24}$ Due to the dispersion of the gain material, the longitudinal mode frequency of an ordinary He-Ne laser is closer to the center frequency than that of the passive cavity. In the case of the Y-shaped cavity laser, the $S$-light and $P$-light share the gain region. As a result, the frequency drift caused by the mode-pulling effect is counteracted, and the influence on the frequency difference drift is relatively small. However, dispersion of the gain material near the center frequency $v_{0}$ of the gain curve will cause the 
frequency difference to fluctuate. We must control the current stability to stabilize the temperature in the gain region and reduce frequency fluctuations caused by dispersion.

For the saturation effect term $\Delta v_{E}^{2}$, in the case of a small signal, the influence of the selfrepulsive term $\Delta v_{\rho}$ can be ignored. Differentiate $\Delta v_{\tau}$ to get

$$
d \Delta v_{\tau}=\tau_{P S} d E_{S}^{2}+E_{S}^{2} d \tau_{P S}-\tau_{S P} d E_{P}^{2}-E_{P}^{2} d \tau_{S P},
$$

This shows that, when the intensity of the $S$-light and $P$-light are approximately equal, the frequency difference drift caused by the saturation effect term $d \Delta v_{\tau}$ is small. In this case, the longitudinal mode of the $S$-light and $P$-light must be separated on both sides of the gain curve.

In short, if we want to use the passive frequency stabilization method to obtain a good frequency difference stabilization in the Y-shaped cavity dual-frequency laser, we must achieve vibration isolation and long-term stability of the temperature and the discharge current. Moreover, we need to keep the intensity of the $S$-light and $P$-light approximately equal. The longitudinal mode of the two lights should be separated on both sides of the gain curve.

\section{Experimental Setup}

To achieve the conditions of frequency stabilization in the theoretical analysis, we designed a precision passive frequency difference stabilization system as shown in Fig. 2. A two-stage thermostat (TST) made from aluminum alloy was designed to precisely control the temperature of the laser. Aluminum allows for good uniform heating and vibration damping. After soaking in concentrated nitric acid, it produces a dense oxide that is not easy to oxidize. To make the laser work in the temperature range corresponding to the lowest expansion coefficient of Zerodur glass, we choose a thermo electric cooler (TEC) as the temperature control actuator. It was evenly glued on the two-stage aluminum box through thermally conductive silica gel to ensure good temperature uniformity. Two digital temperature servo systems with two separate temperature sensors are used to stabilize the TST. The second-level aluminum box serves as a heat sink for the first-level aluminum box, and the third-level aluminum box serves as a heat sink for the TECs. The negative temperature coefficient thermistors are placed near the corresponding TEC. The area near each TEC can be stabilized within $0.6 \mathrm{mK}$, and finally the temperature control accuracy of the inner shield of the TST can reach within $0.6 \mathrm{mK}$. To further suppress the influence of external temperature disturbance on the setup, we use multiple layers of highly reflective aluminum-coated Mylar shields as the thermal insulating layer to cover the TST. It can make the temperature of device more uniform and can effectively reduce the impact of rapid environmental temperature changes on the laser.

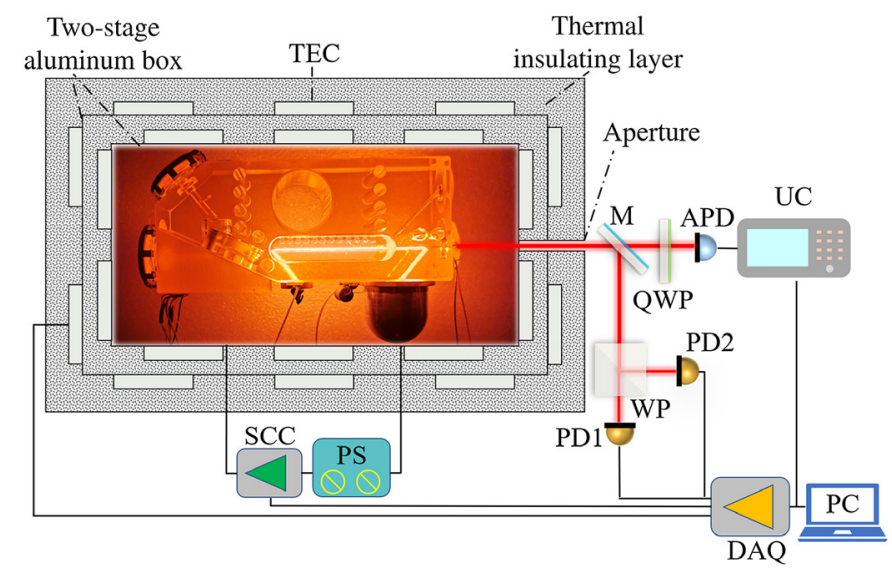

Fig. 2 The experimental setup of frequency difference stabilization using the precision passive control method. 
Two aluminum shields are firmly connected by four thermal insulation gaskets, and the bottom surface of the laser is rigidly mounted on the mid-plane of the TST, which is fixed on the passive vibration isolation platform to maximize the filtering of low frequencies and high-frequency noise, effectively reducing the cavity length changes caused by vertical and horizontal acceleration. In addition, a precision steady current circuit (SCC) was designed to stabilize the current of the $\mathrm{He}-\mathrm{Ne}$ laser within \pm 2 microampere.

The laser beam exits from the small aperture in the middle of the TST. After being split by the beamsplitter M, one beam passes through the quarter-wave plate QWP and is detected by the avalanche photodiode (HAMAMATSU C5658). The $S$-polarized light and $P$-polarized light can be regarded as a circular polarized light after being combined. And the combined light becomes linearly polarized in the same direction after passing through the QWP, which can generate beat frequency. The frequency difference signal displays in the Universal Counter (Agilent 53131A) and is collected by the computer. The other beam is split by a Wollaston prism (WP). The realtime light intensity signals of the $P$-light and $S$-light are received via photodiodes PD1 and PD2, respectively. A computer collects the data and controls the TST and SCC through data acquisition circuit DAQ (NI USB-6366).

The laser in the TST is placed in the laboratory with a temperature of $26^{\circ} \mathrm{C}$. Without any control, after the laser is warmed up for $2 \mathrm{~h}$, the resulting drift curve of the frequency difference $v$, current $I$, and temperature of gain zone surface $T_{S}$ are shown in Fig. 3.

In the $3 \mathrm{~h}$ test, the room temperature is increased $0.1^{\circ} \mathrm{C}$, and the unstable discharge current is changed by $0.06 \mathrm{~mA}$, which causes the surface temperature of the gain zone to change $0.3^{\circ} \mathrm{C}$. The excited current causes the temperature gradient difference between the two sub-cavities and intensifies the dispersion of the gain zone, which enhances the mode pulling effect. Finally, 7.7 $\mathrm{MHz}$ relative frequency difference drift is generated, meaning the floating ratio is $2.36 \%$, which does not meet the measurement stability requirements. To clearly explain the frequency difference drift from the perspective of gain change, we also record the light intensity drift of the $S$-light and $P$-light under the nature state, which is shown in Fig. 4.

In the natural state, the change of light intensity and frequency difference are simultaneous. Because the laser gain $(\mathrm{He}-\mathrm{Ne})$ is common in two-polarization laser mode, an increase in the intensity of one mode will inevitably cause a decrease in the intensity of the other mode. The change trend of intensity between the two lights is always opposite. In the Fig. 4, the noise of the $S$-light intensity is obviously larger than that of the $P$-light because the frequency of the $S$-light is closer to the center frequency of the gain curve. On the one hand, the laser working material exhibits strong dispersion at the center frequency of the curve. On the other hand, the change in the discharge current amplifies the instability of the gain saturation effect. Both make the light intensity near the center frequency of the gain curve more unstable. The above shows that the temperature change and mode-pulling effect caused by the current will greatly affect the frequency difference stability. To find the relationship between temperature change and frequency difference drift, we controlled the current at $2.260 \pm 0.002 \mathrm{~mA}$ and heated the TST from $45^{\circ} \mathrm{C}$ to $63^{\circ} \mathrm{C}$ at a rate of $0.006^{\circ} \mathrm{C}$ per second. The result is shown in Fig. 5 .

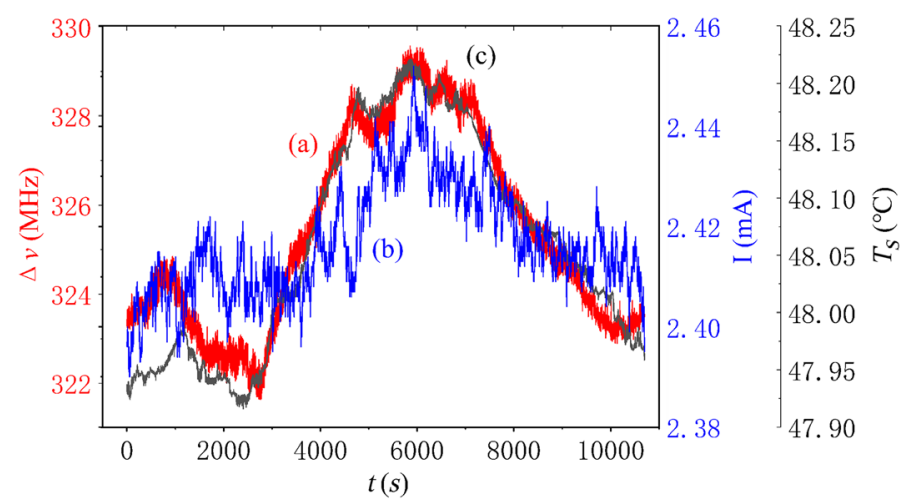

Fig. 3 The drift curve of (a) the frequency difference $\Delta v$ (red); (b) the current I (blue); and (c) the temperature of the gain zone surface $T_{S}$ (black) under the natural state. 


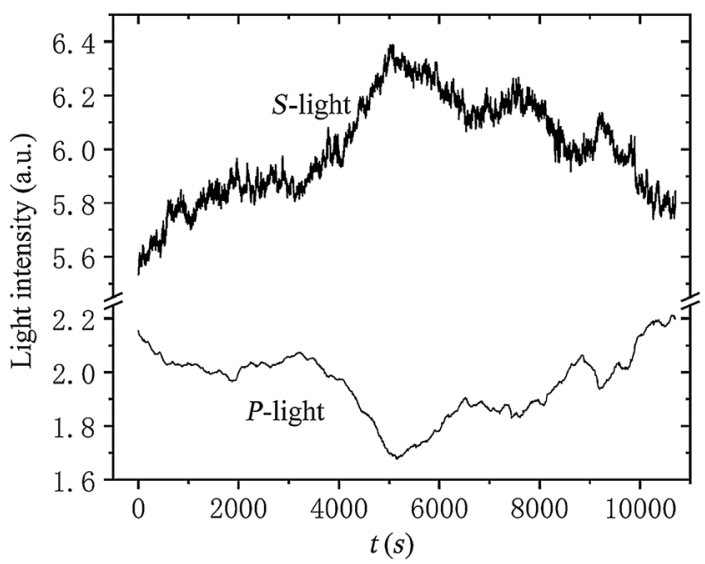

Fig. 4 The light intensity drift of the $S$-light and $P$-light under the natural state.

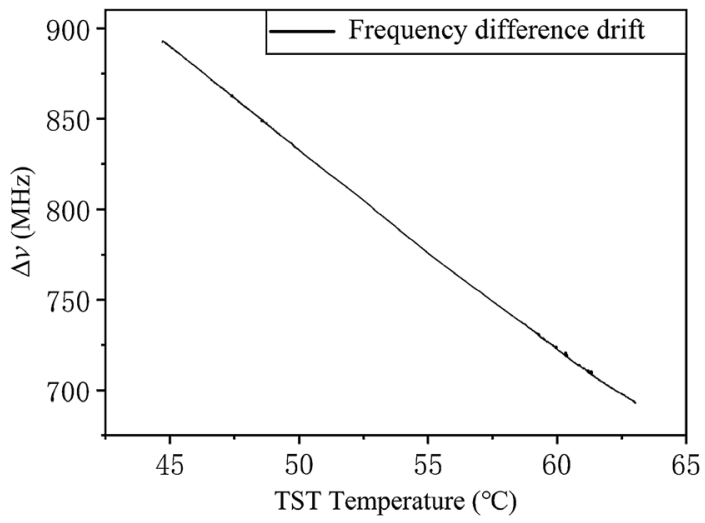

Fig. 5 The curve of frequency difference $\Delta v$ with the TST temperature change.

It can be clearly seen from Fig. 5 that, when the current is stabilized to $2.260 \pm 0.002 \mathrm{~mA}$, the frequency difference and the temperature change show a good linear relationship. The middle and rear parts of the curve have a certain bend due to the delay of heating when the PID control temperature rises to the set value. In the $3000 \mathrm{~s}$ measurement time, the TST temperature change of $18.33^{\circ} \mathrm{C}$ causes the frequency difference drift to be $199.35 \mathrm{MHz}$. Ignoring the influence of other factors, the linear ratio of the temperature to frequency difference drift can be calculated to be $10.88 \mathrm{kHz} / \mathrm{mK}$, which is different from the result calculated by Eq. (3) by $2.46 \mathrm{kHz} / \mathrm{mK}$. This error comes from the mode-pulling effect and the uneven temperature transmission of the two sub-cavities. Moreover, according to the Pearson correlation coefficient, ${ }^{25}$ we calculate the correlation coefficient between the frequency difference and temperature as $r=-0.9998$. This experiment proves that the main factor affecting the frequency difference stability of the Y-shaped cavity dual-frequency laser is the optical path difference between two sub-cavities caused by temperature change. The mode-pulling effect may also play a role. At the same time, changing the TST temperature can be used to tune the frequency difference. Therefore, we need to stabilize the TST temperature and precisely control the laser current to achieve good frequency difference stability.

\section{Results and Discussions}

According to the laser cavity length and resonance conditions, the longitudinal mode spacing is calculated to be $1.456 \mathrm{GHz}$. This means that the frequency difference tuning range of the Yshaped cavity dual-frequency laser is from 0 to $1.456 \mathrm{GHz}$ when the frequency difference lock-in effect is ignored. However, due to the mode repulsion and pulling effects, as the longitudinal 


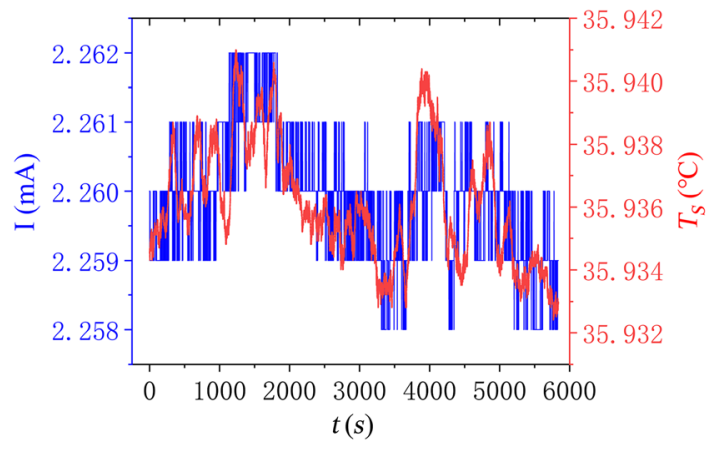

(a)

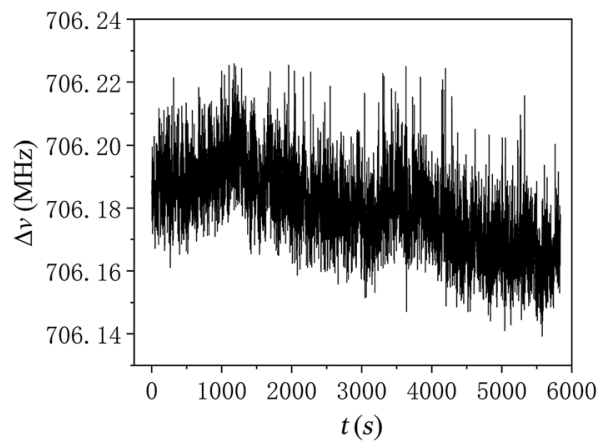

(b)

Fig. 6 The drift curve of (a) the discharge current and gain zone surface temperature. (b) Frequency difference when the TST temperature stabilizes to $24^{\circ} \mathrm{C} \pm 0.4 \mathrm{mK}$.

mode distance gets closer, the frequency difference instability gradually increases. Through the previous theoretical analysis and experimental verification, a good frequency difference stability will be achieved when the vibration is isolated, the cavity temperature and pump current are stable, and the longitudinal mode of the $S$-light and $P$-light are separated on both sides of the gain curve. At a room temperature of $26^{\circ} \mathrm{C}$, the first stage temperature of the TST is stabilized to $24^{\circ} \mathrm{C} \pm 1 \mathrm{mK}$ and the second stage temperature to $24^{\circ} \mathrm{C} \pm 0.4 \mathrm{mK}$ by PID temperature control. We use home-made precision SCC to stabilize the pump current to $2.260 \pm 0.002 \mathrm{~mA}$. The discharge current, gain zone surface temperature, and frequency difference changes are shown in Fig. 6.

It can be seen from Fig. 6(a) that, although the TST temperature change is stabilized within $0.8 \mathrm{mK}$, the surface temperature of the gain zone changes by about $8 \mathrm{mK}$ with the discharge current changes of $0.004 \mathrm{~mA}$. The discharge current increases the temperature of the gain area to $35.9^{\circ} \mathrm{C}$. The frequency difference drift caused by the temperature change is shown in Fig. 6(b). This result is slightly smaller than the linear ratio of the temperature to frequency difference drift of $10.88 \mathrm{kHz} / \mathrm{mK}$ obtained in the Sec. 3 because the TST temperature is $11.9^{\circ} \mathrm{C}$ lower than the gain zone temperature. The temperature difference of $8 \mathrm{mK}$ is quickly suppressed during the diffusion process and does not evenly affect the entire cavity. The experimental result proves the validity of the theoretical analysis.

When the precision passive control is implemented, the TST temperature is stabilized to $24^{\circ} \mathrm{C} \pm 0.4 \mathrm{mK}$, and the current is stabilized to $2.260 \pm 0.002 \mathrm{~mA}$. The frequency difference drift is reduced to below $80 \mathrm{kHz}$, which means that the drift rate does not exceed $0.011 \%$ (variation value divided by the average value). This is much smaller than the natural drift, which is 7.7 MHz relative frequency difference drift, meaning the floating ratio is $2.36 \%$. The square root of the Allan variance of the frequency-difference stability is $9 \times 10^{-11}$, and the square root of the Allan variance of the laser absolute frequency stability is $8.2 \times 10^{-10}$. The results show that the device can reduce the frequency difference drift rate of the Y-shaped cavity dual-frequency laser by two orders of magnitude, which meets the needs of industrial testing. Moreover, the vibration isolation and temperature and current control decrease the external influence on the laser and improve its environmental adaptability.

In previous research, because of the clever structure design in the Y-shaped cavity dualfrequency laser, a 26 to $625 \mathrm{MHz}$ frequency difference range was achieved. ${ }^{8}$ Also, in this case, a $10^{-5}$ to $10^{-6}$ acceleration measurement resolution $\left( \pm 5 \mathrm{~g}\right.$ measurement range) and $10^{-5} \mathrm{~N}$ force measurement resolution can be expected. ${ }^{12,13}$ In 2015 , our group discovered a method to weaken the frequency difference lock-in phenomenon through the transverse magnetic field, which further broadened the frequency difference range. ${ }^{14}$ At present, we have expanded the frequency difference output range to 0 to $1.456 \mathrm{GHz}$ when the frequency difference lock-in effect was ignored. The relative frequency difference drift rate is controlled within $0.011 \%$. It is reasonable to believe that the range and accuracy of acceleration and force measurement will be further improved. The Y-shaped cavity dual-frequency laser can become an excellent light source for precise physical quantities measurement, synthetic wavelength, and heterodyne interference. 


\section{Conclusion}

In this paper, theoretical analysis and experimental verification of the main factors affecting the frequency difference stability of the Y-shaped cavity dual-frequency laser are carried out. To improve the frequency difference stability, a precise passive control of the temperature and current schemes is proposed. It can achieve vibration isolation and long-term stability of the temperature and the discharge current, keep the intensity of the $S$-light and $P$-light approximately equal, and separate the longitudinal mode of the two lights on both sides of the gain curve. The temperature of the TST can be stabilized to $24^{\circ} \mathrm{C} \pm 0.4 \mathrm{mK}$ by a PID temperature control. The pump current can be controlled to $2.260 \pm 0.002 \mathrm{~mA}$. In this case, the relative frequency difference in $706.18 \mathrm{MHz}$ is stabilized to within $80 \mathrm{kHz}$, and the relative frequency difference drift rate is $0.011 \%$. This shows an improvement of two orders of magnitude over the stress-induced birefringence closed-loop control method. ${ }^{17}$ Compared with the natural drift, this method can achieve long-term stability of the frequency difference. It is easy to implement and completely suitable for industrial and laboratory precision measurement fields. Also, it can be applied to other laser systems using different frequency stabilization techniques, which is of great significance in improving the accuracy of acceleration and force measurement. Currently, the frequency difference stability is limited by the temperature fluctuation in the gain zone. Future implementations of the system may include adopting an ultralow-expansion glass cavity and further reducing current drift, thereby increasing the frequency difference stability.

\section{Acknowledgments}

The authors wish to thank Tengfang Kuang and Zichao Wang for their help in reviewing and modifying this paper. The authors declare no conflicts of interest.

\section{References}

1. R. B. Suhoke, "Laser optical methods for measurement of velocity and vibration," Proc. SPIE 0741, 196 (1987).

2. W. Holzapfel and M. Finnemann, "High-resolution force sensing by a diodepumped Nd: YAG laser," Opt. Lett. 18, 2062-2064 (1993).

3. V. Molebny, G. Kamerman, and O. Steinvall, "Laser radar: from early history to new trends," Proc. SPIE 7835, 783502 (2010).

4. R. Diaz, S. Chan, and J. Liu, "Lidar detection using a dual-frequency source," Opt. Lett. 31, 3600-3602 (2006).

5. J. Li, H. Niu, and Y. X. Niu, "Laser feedback interferometry and applications: a review," Opt. Eng. 56(5), 050901 (2017).

6. Z. Zeng et al., "High density fringes and phase behavior in birefringence dual frequency laser with multiple feedback," Opt. Express 20, 4747-4752 (2012).

7. X. Wan et al., "Influence of optical feedback on the longitudinal mode stability of microchip Nd:YAG lasers," Opt. Eng. 44(10), 104204 (2005).

8. G. Xiao et al., "A novel active optical approach for acceleration measurement based on a Y-shaped cavity dual-frequency laser," Opt. Laser Technol. 44, 344-348 (2012).

9. P. Dumont et al., "Low-noise dual-frequency laser for compact Cs atomic clocks," J. Lightwave Technol. 32, 3817-3823 (2014).

10. C. H. Cheng et al., "Dual-frequency laser Doppler velocimeter for speckle noise reduction and coherence enhancement," Opt. Express 20(18), 20255-20265 (2012).

11. J. Chen et al., "Self-mixing birefringent dual-frequency laser Doppler velocimeter," Opt. Express 25, 560-572 (2017).

12. G. Xiao, X. Long, and B. Zhang, "A novel orthogonal polarized dual-frequency laser using a Y-shaped cavity," Opt. Laser Technol. 43, 1314-1317 (2011).

13. G. Xiao et al., "Precise force measurement method by a Y-shaped cavity dual-frequency laser," Chin. Opt. Lett. 9, 101201 (2011).

14. G. Xiao et al., "Frequency difference lock-in phenomenon's weakening by transverse magnetic field in Y-shaped cavity dual-frequency laser," Chin. Opt. Lett. 13, 111405 (2015). 
Zeng, Zhang and Xiao: Frequency difference stabilization in Y-shaped cavity dual-frequency laser...

15. C. Sternkopf and E. Manske, "Digital frequency offset-locked He-Ne laser system with high beat frequency stability, narrow optical linewidth and optical fibre output," Meas. Sci. Technol. 29, 064013 (2018).

16. S. Subhankar et al., "Microcontroller based scanning transfer cavitylock for long-term laser frequency stabilization,” Rev. Sci. Instrum. 90, 043115 (2019).

17. J. Li, Y. Niu, and H. Niu, "Frequency difference stabilization in dual-frequency laser by stress-induced birefringence closed-loop control," Appl. Opt. 55(16), 4357-4361 (2016).

18. J. C. Tsai, "Stabilizing laser frequencies for metrology applications," Proc. SPIE 741, 186-195 (1987).

19. M. Schiemangk et al., "Accurate frequency noise measurement of free-running lasers," Appl. Opt. 53(30), 7138-7143 (2014).

20. D. Yoshitomi and K. Torizuka, "Long-term stable passive synchronization between twocolor mode-locked lasers with the aid of temperature stabilization," Opt. Express 22(4), 4091-4097 (2014).

21. S. Häfner et al., " $8 \times 10^{-17}$ fractional laser frequency instability with a long room-temperature cavity," Opt. Lett. 40(9), 2112-2115 (2015).

22. J. Alnis et al., "Subhertz linewidth diode lasers by stabilization to vibrationally and thermally compensated ultralow-expansion glass Fabry-Pérot cavities," Phys Rev A 77, 053809 (2008).

23. W. E. Lamb, Jr., "Theory of an optical maser," Phys. Rev. 134(6A), A1429 (1964).

24. A. Lindberg, "Mode frequency pulling in He-Ne lasers," Am. J. Phys. 67, 350-353 (1999).

25. J. L. Rodgers and W. A. Nicewander, "Thirteen ways to look at the correlation coefficient," Am. Stat. 42(1), 59-66 (1988).

Xiaofei Zeng is an MS candidate at the National University of Defense Technology. He received his BS degree in physics from the National University of Defense Technology in 2015. His current research interests include gas lasers and optical traps.

Bin Zhang received his MS degree from the National University of Defense Technology in 1993. Currently, he is a professor at the National University of Defense Technology.

Guangzong Xiao received his PhD from the National University of Defense Technology in 2011. Currently, he is a professor at the National University of Defense Technology. His research focuses on gas lasers and optical traps.

Jianping Liu received his MS degree from the Beijing Institute of Technology in 2003. Currently, he is a professor at the National University of Defense Technology.

Hongchang Zhao received his MS degree from the National University of Defense Technology in 2001. Currently, he is a professor at the National University of Defense Technology. 